\title{
ON DEGREE OF APPROXIMATION BY PRODUCT MEANS OF CONJUGATE SERIES OF A FOURIER SERIES
}

\author{
S. K. Paikray ${ }^{1}$, R. K. Jati ${ }^{2}$, N. C. Sahoo ${ }^{3}$, U.K.Misra ${ }^{4}$ \\ ${ }^{1}$ P.G.Department of Mathematics, Ravenshaw University, Cuttack-753003, Odisha,India \\ ${ }^{2}$ Department of Mathematics, DRIEMS, Tangi,Cuttack, Odisha, India \\ ${ }^{3}$ Department of Mathematics,S.B. Women's College(Auto), Cuttack, Odisha, India \\ ${ }^{4}$ Science and Technology, Pallur Hills, Golanthara-761008, Odisha, India
}

Keywords: Degree of Approximation $f \in \operatorname{Lip}(\alpha, r)$ class of function, $(E, q)$ mean, $\left(\bar{N}, p_{n}\right)$ mean, $(E, q)\left(\bar{N}, p_{n}\right)$ product mean, Fourier series, Conjugate of the Fourier series, Lebesgue integral

\begin{abstract}
In this paper a theorem on degree of approximation of a function $f \in \operatorname{Lip}(\alpha, r)$ by product summability $\left.(E, q) \bar{N}, p_{n}\right)$ of conjugate series of Fourier series associated with $\mathrm{f}$ has been established.
\end{abstract}

\section{Introduction}

Let $\sum \mathrm{a}_{\mathrm{n}}$ be a given infinite series with the sequence of partial sums $\left\{s_{n}\right\}$. Let $\left\{p_{n}\right\}$ be a sequence of positive real numbers such that

$$
P_{n}=\sum_{v=0}^{n} p_{v} \rightarrow \infty, \quad \text { as } \quad n \rightarrow \infty,\left(P_{-i}=p_{-i}=0, i \geq 0\right) .
$$

The sequence -to-sequence transformation

$$
t_{n}=\frac{1}{P_{n}} \sum_{v=0}^{n} p_{v} s_{v}
$$

defines the sequence $\{\operatorname{tn}\}$ of the $\left.\overline{(N}, p_{n}\right)$-mean of the sequence $\left\{s_{n}\right\}$ generated by the sequence of coefficient $\left\{p_{n}\right\}$. If

$$
t_{n} \rightarrow s \quad \text {,as } n \rightarrow \infty
$$

then the series $\Sigma$ an is said to be $\left(N, \bar{p}_{n}\right)$ summable to s.

The conditions for regularity of $\left(N, p_{n}\right)$ - summability are easily seen to be[1]

$$
\left\{\begin{array}{l}
\text { (i) } P_{n} \rightarrow \infty \text {, as } n \rightarrow \infty . \\
\text { (ii) } \sum_{i=0}^{n} p_{i} \leq C\left|P_{n}\right| \text {, as } n \rightarrow \infty .
\end{array}\right.
$$

The sequence -to-sequence transformation, [1]

$$
T_{n}=\frac{1}{(1+q)^{n}} \sum_{v=0}^{n}\left(\begin{array}{l}
n \\
v
\end{array}\right) q^{n-v} s_{v}
$$

defines the sequence $\left\{T_{n}\right\}$ of the $(E, q)$ mean of the sequence $\left\{s_{n}\right\}$. If

$$
T_{n} \rightarrow s, \text { as } n \rightarrow \infty
$$

then the series $\sum a_{n}$ is said to be $(E, q)$ summable to $s$. 
Clearly $(E, q)$ method is regular. Further, the $(E, q)$ transform of the $\left(N, p_{n}\right)$ transform of $\left\{\mathrm{s}_{\mathrm{n}}\right\}$ is defined by

$$
\begin{aligned}
\tau_{n} & =\frac{1}{(1+q)^{n}} \sum_{k=0}^{n}\left(\begin{array}{l}
n \\
k
\end{array}\right) q^{n-k} t_{k} \\
& =\frac{1}{(1+q)^{n}} \sum_{k=0}^{n}\left(\begin{array}{l}
n \\
k
\end{array}\right) q^{n-k}\left\{\frac{1}{P_{k}} \sum_{v=0}^{k} p_{v} s_{v}\right\}
\end{aligned}
$$

If

$$
\tau_{n} \rightarrow s, \text { as } n \rightarrow \infty
$$

then $\sum a_{n}$ is said to be $(E, q)\left(N, p_{n}\right)$-summable to $s$.

Let $f(t)$ be a periodic function with period $2 \pi$ and L-integrable over $(-\pi, \pi)$. The Fourier series associated with $f$ at any point $\mathrm{x}$ is defined by

$$
f(x) \sim \frac{a_{0}}{2}+\sum_{n=1}^{\infty}\left(a_{n} \cos n x+b_{n} \sin n x\right) \equiv \sum_{n=0}^{\infty} A_{n}(x)
$$

and the conjugate series of the Fourier series (1.9) is

$$
\sum_{n=1}^{\infty}\left(b_{n} \cos n x-a_{n} \sin n x\right) \equiv \sum_{n=1}^{\infty} B_{n}(x)
$$

Let $\overline{S_{n}}(\mathrm{f} ; \mathrm{x})$ be the $\mathrm{n}$-th partial sum of (1.10). The L $\infty$-norm of a function $f: R \rightarrow R$ is defined by

$$
\|f\|_{\infty}=\sup \{f(x) \mid: x \in R\}
$$

and the $L_{v}$-norm is defined by

$$
\|f\|_{v}=\left(\int_{0}^{2 \pi}|f(x)|^{v}\right)^{\frac{1}{v}}, v \geq 1
$$

The degree of approximation of a function $f: R \rightarrow R$ by a trigonometric polynomial $P_{n}(x)$ of degree $n$ under norm.\|\|$_{\infty}$ is defined by [5].

$$
\left\|P_{n}-f\right\|_{\infty}=\sup \left\{\left|p_{n}(x)-f(x)\right|: x \in R\right\}
$$

and the degree of approximation $E_{n}(f)$ or a tunction $j \in L_{v}$ is given by

$$
E_{n}(f)=\min _{P}\left\|P_{n}-f\right\|_{0}
$$

A function $\mathrm{f}$ is said to satisfy Lipschitz condition (here after we write $f \in \operatorname{Lipa}$ ) if

$$
|f(x+t)-f(x)|=O\left(|t|^{\alpha}\right), 0<\alpha \leq 1
$$

and $f(x) \in \operatorname{Lip}(a, r)$, for $0 \leq x \leq 2 \pi$, if

$$
\left(\int_{0}^{2 \pi}|f(x+t)-f(x)|^{r} d x\right)^{\frac{1}{r}}=O\left(|t|^{\alpha}\right), 0<\alpha \leq 1, r \geq 1, t>0
$$


We use the following notation throughout this paper :

$$
\psi(t)=\frac{1}{2}\{f(x+t)-f(x-t)\}
$$

and

$$
\overline{K_{n}}(t)=\frac{1}{\pi(1+q)^{n}} \sum_{k=0}^{n}\left(\begin{array}{l}
n \\
k
\end{array}\right) q^{n-k}\left\{\frac{1}{P_{k}} \sum_{v=0}^{k} p_{v} \frac{\cos \frac{t}{2}-\cos \left(v+\frac{1}{2}\right) t}{\sin \frac{t}{2}}\right\}
$$

Further, the method $(E, q)\left(N, \bar{p}_{n}\right)$ is assumed to be regular .

\section{Known Theorem}

Dealing with The degree of approximation by the product $(\mathrm{E}, \mathrm{q})(\mathrm{C}, 1)$-mean of Fourier series, Nigam et al [3] proved the following theorem:

Theorem 2.1. If a function $f$ is $2 \pi$ - periodic and belonging to class Lipa, then its degree of approximation by $(E, q)(C, 1)$ summability mean on its Fourier series $\sum_{n=0}^{\infty} A_{n}(t)$ is given by $\left\|E_{n}^{q} C_{n}^{1}-f\right\|_{\infty}=O\left(\frac{1}{(n+1)^{\alpha}}\right), 0<\alpha<1 \quad$ where $E_{n}^{q} C_{n}^{1}$ represents the $(E, q)$ transform of $(\mathrm{C}, 1)$ transform of $s_{n}(f ; x)$.

Recently, Misra et al [2] proved the following theorem using $(E, q)\left(N, p_{n}\right)$ mean of conjugate series of the Fourier series :

Theorem 2.2. If $f$ be $2 \pi$ - Periodic function of class Lipa, then degree of approximation by the product $\left.(E, q) \bar{N}, p_{n}\right)$ summability means of the conjugate series (1.10) of Fourier series (1.9) is given $\left\|\tau_{n}-f\right\|_{\infty}=O\left(\frac{1}{(n+1)^{\alpha}}\right), 0<\alpha<1$ where $\tau_{\mathrm{n}}$ is as defined in 1.7).

\section{Main theorem}

In this paper, we have proved a theorem on degree of approximation by the product mean $(E$, $q)(N, p)$ of conjugate series of Fourier series of a function of class $\operatorname{Lip}(a, r)$. We prove:

Theorem.3.1. If $f$ be $2 \pi$-Periodic function of class Lipa, then degree of approximation by the product $\left.(E, q) \bar{N}, p_{n}\right)$ summability means of the conjugate series of Fourier series (1.10) is given by

$$
\left\|\tau_{n}-f\right\|_{\infty}=O\left(\frac{1}{(n+1)^{\alpha-1 / r}}\right), 0<\alpha<1
$$

\section{Required Lemmas}

where $\tau$ is as defined in (1.7).

We require the following Lemmas to prove the theorem.

\section{Lemma 4.1}

$$
\left|\overline{K_{n}}(t)\right|=O(n) \quad, 0 \leq t \leq \frac{1}{n+1}
$$




\section{Proof.}

For $0 \leq t \leq \frac{1}{n+1}$ we have $\sin \mathrm{nt} \leq \mathrm{nsin} \mathrm{t}$ then

$$
\begin{aligned}
\left|\overline{K_{n}}(t)\right| & =\frac{1}{\pi(1+q)^{n}}\left|\sum_{k=0}^{n}\left(\begin{array}{l}
n \\
k
\end{array}\right) q^{n-k}\left\{\frac{1}{P_{k}} \sum_{v=0}^{k} p_{v} \frac{\cos \frac{t}{2}-\cos \left(v+\frac{1}{2}\right) t}{\sin \frac{t}{2}}\right\}\right| \\
& \leq \frac{1}{\pi(1+q)^{n}} \mid \sum_{k=0}^{n}\left(\begin{array}{l}
n \\
k
\end{array}\right) q^{n-k}\left\{\frac{1}{P_{k}} \sum_{v=0}^{k} p_{v} \frac{\cos \frac{t}{2}-\cos v t \cdot \cos \frac{t}{2}+\sin v t \cdot \sin \frac{t}{2}}{\sin \frac{t}{2}}\right\} \\
& \leq \frac{1}{\pi(1+q)^{n}} \mid \sum_{k=0}^{n}\left(\begin{array}{l}
n \\
k
\end{array}\right) q^{n-k}\left\{\frac{1}{P_{k}} \sum_{v=0}^{k} p_{v}\left(\frac{\cos \frac{t}{2}\left(2 \sin 2 v \frac{t}{2}\right)}{\sin \frac{t}{2}+\sin v t}\right) \mid\right\} \\
& \leq \frac{1}{\pi(1+q)^{n}} \mid \sum_{k=0}^{n}\left(\begin{array}{l}
n \\
k
\end{array}\right) q^{n-k}\left\{\frac{1}{P_{k}} \sum_{v=0}^{k} p_{v}\left(O\left(2 \sin v \frac{t}{2} \sin v \frac{t}{2}\right)+v \sin t\right)\right\} \\
& \leq \frac{1}{\pi(1+q)^{n}}\left|\sum_{k=0}^{n}\left(\begin{array}{l}
n \\
k
\end{array}\right) q^{n-k}\left\{\frac{1}{P_{k}} \sum_{v=0}^{k} p_{v}(O(v)+O(v))\right\}\right| \\
& \leq \frac{1}{\pi(1+q)^{n}}\left|\sum_{k=0}^{n}\left(\begin{array}{l}
n \\
k
\end{array}\right) q^{n-k} \frac{O(k)}{P_{k}} \sum_{v=0}^{k} p_{v}\right| \\
& =O(n)
\end{aligned}
$$

This proves the lemma.

\section{Lemma 4.2}

$$
\left|\overline{K_{n}}(t)\right|=O\left(\frac{1}{t}\right), \text { for } \frac{1}{n+1} \leq t \leq \pi
$$

Proof.

For $\frac{1}{n+1} \leq t \leq \pi$, by Jordan's lemma, we have $\sin \left(\frac{t}{2}\right) \geq \frac{t}{\pi}$

Then

$$
\begin{aligned}
& \left|\overline{K_{n}}(t)\right|=\frac{1}{\pi(1+q)^{n}}\left|\sum_{k=0}^{n}\left(\begin{array}{l}
n \\
k
\end{array}\right) q^{n-k}\left\{\frac{1}{P_{k}} \sum_{v=0}^{k} p_{v} \frac{\cos \frac{t}{2}-\cos \left(v+\frac{1}{2}\right)}{\sin \frac{t}{2}}\right\}\right| \\
& =\frac{1}{\pi(1+q)^{n}}\left|\sum_{k=0}^{n}\left(\begin{array}{l}
n \\
k
\end{array}\right) q^{n-k}\left\{\frac{1}{P_{k}} \sum_{v=0}^{k} p_{v} \frac{\cos \frac{t}{2}-\cos v \frac{t}{2} \cdot \cos \frac{t}{2}+\sin v \frac{t}{2} \cdot \sin \frac{t}{2}}{\sin \frac{t}{2}}\right\}\right|
\end{aligned}
$$




$$
\begin{aligned}
& \leq \frac{1}{\pi(1+q)^{n}} \mid \sum_{k=0}^{n}\left(\begin{array}{l}
n \\
k
\end{array}\right) q^{n-k}\left\{\frac{1}{P_{k}} \sum_{v=0}^{k} \frac{\pi}{2 t} p_{v}\left(\cos \frac{t}{2}\left(2 \sin ^{2} v \frac{t}{2}\right)+\sin v \frac{t}{2} \cdot \sin \frac{t}{2}\right)\right\} \\
& \leq \frac{\pi}{2 \pi(1+q)^{n} t}\left|\sum_{k=0}^{n}\left(\begin{array}{l}
n \\
k
\end{array}\right) q^{n-k}\left\{\frac{1}{P_{k}} \sum_{v=0}^{k} p_{v}\right\}=\frac{1}{2(1+q)^{n} t}\right| \sum_{k=0}^{n}\left(\begin{array}{l}
n \\
k
\end{array}\right) q^{n-k}\left\{\frac{1}{P_{k}} \sum_{v=0}^{k} p_{v}\right\} \mid \\
& =\frac{1}{2(1+q)^{n} t}\left|\sum_{k=0}^{n}\left(\begin{array}{l}
n \\
k
\end{array}\right) q^{n-k}\right| \\
& =O\left(\frac{1}{t}\right) .
\end{aligned}
$$

This proves the lemma.

\section{Proof of theorem 3.1}

Using Riemann -Lebesgue theorem, we have for the n-th partial sum $\left.s_{n} \overline{(\mathrm{f}} ; \mathrm{x}\right)$ of the conjugate Fourier series (1.10) of $f(x)$,following Titchmarch [4]

$$
\overline{s_{n}}(f ; x)-f(x)=\frac{2}{\pi} \int_{0}^{\pi} \psi(t) \overline{K_{n}} d t
$$

the $(N, p n)$ transform of $\left.s_{n} \overline{(} f ; x\right)$ using $(1.2)$ is given by

$$
t_{n}-f(x)=\frac{2}{\pi P_{n}} \int_{0}^{\pi} \psi(t) \sum_{k=0}^{n} p_{k} \frac{\cos \frac{t}{2}-\sin \left(n+\frac{1}{2}\right) t}{2 \sin \left(\frac{t}{2}\right)} d t
$$

denoting the $(E, q)(N, p n)$ transform of $\overline{s_{n}}(f ; x)$ by $\tau_{\mathrm{n}}$, we have

$$
\begin{aligned}
\left\|\tau_{n}-f\right\| & =\frac{2}{\pi(1+q)^{n}} \int_{0}^{\pi} \psi(t) \sum_{k=0}^{n}\left(\begin{array}{l}
n \\
k
\end{array}\right) q^{n-k}\left\{\frac{1}{P_{k}} \sum_{v=0}^{k} p_{v} \frac{\cos \frac{t}{2}-\sin \left(v+\frac{1}{2}\right) t}{2 \sin \left(\frac{t}{2}\right)}\right\} d t \\
& =\int_{0}^{\pi} \psi(t) \overline{K_{n}}(t) d t \\
& =\left\{\int_{0}^{\frac{1}{n+1}+\int_{1}^{\pi}}\right\} \psi(t) \overline{K_{n}}(t) d t \\
=\mathrm{I}_{1}+I_{2}, \text { say } &
\end{aligned}
$$


Now

$$
\begin{aligned}
& \left|I_{1}\right|=\frac{2}{\pi(1+q)^{n}}\left|\int_{0}^{1 / n+1} \psi(t) \sum_{k=0}^{n}\left(\begin{array}{l}
n \\
k
\end{array}\right) q^{n-k}\left\{\frac{1}{P_{k}} \sum_{v=0}^{k} p_{v} \frac{\cos \frac{t}{2}-\cos \left(v+\frac{1}{2}\right) t}{2 \sin \frac{t}{2}}\right\} d t\right| \\
& \leq\left|\int_{0}^{\frac{1}{n+1}} \psi(t) \overline{K_{n}}(t) d t\right| \\
& =\left(\int_{0}^{\frac{1}{n+1}}(\psi(t))^{r} d t\right)^{\frac{1}{r}}\left(\int_{0}^{\frac{1}{n+1}}\left(\bar{K}_{n}(t)\right)^{s} d t\right)^{\frac{1}{s}} \text {, using Holder's inequality } \\
& \leq O\left(\frac{1}{(n+1)^{\alpha}}\right)\left(\int_{0}^{\frac{1}{n+1}} n^{s} d t\right)^{\frac{1}{s}} \quad \text { using Lemma } 4.1 \\
& =O\left(\frac{1}{(n+1)^{\alpha}}\right)\left(\frac{n^{s}}{s+1}\right)^{\frac{1}{s}} \\
& =O\left(\frac{n}{(n+1)^{\alpha+\frac{1}{s}}}\right) . \\
& =O\left(\frac{1}{(n+1)^{\alpha+\frac{1}{s}-1}}\right)=O\left(\frac{1}{(n+1)^{\alpha-\frac{1}{r}}}\right)
\end{aligned}
$$

Next

$$
\begin{aligned}
\left|I_{2}\right| \leq & \left(\int_{\frac{1}{n+1}}^{\pi}(\psi(t))^{r} d t\right)^{\frac{1}{r}}\left(\int_{\frac{1}{n+1}}^{\pi}\left(\overline{K_{n}}(t)\right)^{s} d t\right)^{\frac{1}{s}} \text { using Holder's inequality } \\
& \leq O\left(\frac{1}{(n+1)^{\alpha}}\right)\left(\int_{\frac{1}{n+1}}^{\pi}\left(\frac{1}{t}\right)^{s} d t\right)^{\frac{1}{s}} \text { using Lemma 4.2 } \\
& =O\left(\frac{1}{(n+1)^{\alpha}}\right)\left(\frac{1}{n+1}\right)^{\frac{1-s}{s}-} \\
& =O\left(\frac{1}{(n+1)^{\alpha-\frac{1}{r}}}\right)
\end{aligned}
$$


Then from (5.2) and (5.3), we have

$$
\left|\tau_{n}-f(x)\right|=O\left(\frac{1}{(n+1)^{\alpha-\frac{1}{r}}}\right) \text {, for } 0<\alpha<1, r \geq 1 \text {. }
$$

Hence,

$$
\left\|\tau_{n}-f(x)\right\|_{\infty}=\sup _{-\pi<x<\pi}\left|\tau_{n}-f(x)\right|=O\left(\frac{1}{(n+1)^{\alpha-\frac{1}{r}}}\right), 0<\alpha<1, r \geq 1
$$

This completes the proof of the theorem.

\section{References}

[1] Hardy, G.H: Divergent series, First edition, Oxford University press 70(19).

[2] Misra, U.K., Misra, M., Padhy, B.P. and Buxi, S.K: On degree of approximation by product means of conjugate series of Fourier series", International Jour. of Math. Sciences, and Engineering Applications, ISSN 0973 - 9424, Vol.6 No.122 (Jan. 2012), pp 363 - 370.

[3] Nigam, H.K and Ajay Sharma: On degree of Approximation by product means, Ultra Scientist of Physical Sciences, Vol.22 (3) M, 889-894, (2010).

[4] Titchmarch, E.C: The theory of functions, oxford university press, p.p402-403(1939).

[5] Zggmund, A : Trigonometric Series, second Edition ,Vol.I , Cambridge University press , Cambridge, (1959). 